\title{
Predictors of IVF/ICSI success following treatment of endometriosis as the cause of primary infertility
}

\author{
Jovan S. Bila ${ }^{1,2}$, Snezana Vidakovic ${ }^{1,2}$, Svetlana Spremovic Radjenovic ${ }^{1,2}$, \\ Milan Dokic ${ }^{1,2}$, Lela Surlan', Radmila Sparic ${ }^{1,2}$ \\ ${ }^{1}$ Clinic of Obstetrics and Gynecology, Clinical Center of Serbia, Belgrade, Serbia \\ ${ }^{2}$ Faculty of Medicine, University of Belgrade, Belgrade, Serbia
}

\begin{abstract}
Objectives: Treatment of endometriosis prior to IVF/ICSI could be followed by the significant reduction of ovarian reserve. The aim is to identify potential markers of the IVF/ICSI outcome in patients with endometriosis associated infertility and to evaluate their clinical significance.

Material and methods: The prospective cohort study included 73 patients with primary infertility caused by endometriosis that were subjected to 77 IVF/ICSI cycles. Patients were classified into two groups. In the first group some type of treatment had previously been applied, and in the second group patients were immediately subjected to the IVF/ICSI procedures.

Results: When pregnancy was achieved, there were significantly more patients under 35 years of age, more patients with primary infertility duration up to 3 years, and more patients with endometriosis that was previously treated $(77.4 \%)$ $(p<0.039)$. In the cases of the successful outcome Endometriosis Fertility Index $>7$, lower basal FSH and FSH/LH ratio were found, as well as significantly higher basal E2, basal P4 and AMH. Significantly lower doses of gonadotropins were needed in cases of the successful outcome, and long protocol with agonists was more frequently used. Multivariate logistic regression analysis showed that previous therapy of endometriosis, $\mathrm{P} 4 \geq 0.7 \mathrm{ng} / \mathrm{mL}, \mathrm{AMH} \geq 0.9 \mathrm{ng} / \mathrm{mL}, \mathrm{A}$ class of embryos, and the use of long protocol with agonists were predictors of the successful IVF/ICSI outcome.

Conclusions: Therapy for endometriosis, AMH and P4 levels appeared to be predictors for the successful IVF/ICSI outcome and the use of long protocol with agonists could be advised in these cycles.
\end{abstract}

Key words: endometriosis, IVF/ICSI, ovarian reserve, AMH, long protocol

Ginekologia Polska 2018; 89, 5: 240-248

\section{INTRODUCTION}

The link between endometriosis and infertility is usually explained by the simultaneous effect of multiple mechanisms, such as immune factors, disturbed folliculogenesis and ovulation, oocyte degeneration and apoptosis, compromised fertilization and abnormal embryogenesis [1-3]. Surgical treatment of endometriosis prior to IVF is common, but could be followed by the significant reduction of ovarian reserve which has a negative impact on fertility $[4,5]$.

Many studies suggest that women with endometriosis have lower ovarian reserve and higher basal FSH level than women of the same age who do not have endometriosis [6].
Serum anti-Müllerian hormone (AMH) level accurately reflects the ovarian reserve, which may be lower in women with endometriosis, and can predict the ovarian response to controlled ovarian hyperstimulation $(\mathrm{COH})$ in these patients $[7,8]$. Endometriosis fertility index or EFI was proposed for the fertility assessment after endometriosis treatment [9]. This index includes anamnestic parameters such as age, duration of infertility, parity, as well as anatomical and functional assessment of the disease severity.

The aim was to identify potential markers of the successful IVF/ICSI outcome in patients with endometriosis associated infertility and to evaluate their clinical significance. 


\section{MATERIAL AND METHODS \\ Patients}

The prospective cohort study was conducted at the tertiary institution for a period of 5 years, and included 73 patients with primary infertility caused by endometriosis that were subjected to 77 IVF/ICSI cycles. The study was approved by the Ethics committee of the Medical faculty University of Belgrade, Serbia, and all patients gave informative consent. Inclusion criteria were: age up to 40 years, primary infertility lasting for more than a year and caused by endometriosis, the absence of associated infertility factors, body mass index (BMI) less than $30 \mathrm{~kg} / \mathrm{m}^{2}$, regular cycles 24-35 days, or previously diagnosed endometriosis independently to infertility treatment. Patients were classified into two groups. In the first group (Group I) some kind of endometriosis treatment had previously been applied before the introduction to the IVF/ICSI, and in the second group (Group II) patients were immediately subjected to the IVF/ICSI procedures. The Group I consisted of patients diagnosed with endometriosis on surgical treatment that could have been followed by the medical treatment. Therefore in the study group we formed two subgroups of patients, first subgroup (A) with combined surgical and medical treatment and second subgroup of patients (B) who were only surgicaly treated. The Group II consisted of patients with endometriosis that were not treated and those patients were immediately introduced to the IVF/ICSI. In these cases endometriosis was diagnosed previously and independently to infertilty treatment or presence of endometrioma was confirmed on ultrasound. Age, body mass index (BMI), endometriosis fertility index (EFI), endometrioma presence, diameter and laterality, as well as the ASRM stage were determined for all patients.

\section{Therapy}

Surgical treatment was applied in patients with symptoms such as pelvic pain and dysmenorrhoea, and/or with severe forms of endometriosis or presence of endometrioma $>3 \mathrm{~cm}$, or bilateral endometriomas $>3 \mathrm{~cm}$. Surgical treatment involved laparoscopic approach, excision of the endometriotic capsule with stripping technique and achieving hemostasis using bipolar forceps. Depending on the disease severity, excision and vaporization of other endometriotic foci were made as well.

Combined surgical and medicament treatment invoIved the introduction of gonadotropin-releasing hormone (GnRH) agonists every 28 days for 3-6 months immediately after the surgical treatment and the histopathological verification of the endometriosis. Additional medical therapy was introduced if the patients had more pronounced symptoms before surgery, and/or had more severe form of endometriosis with dissemination that was verified on operation.
In the Group II presence of endometriomas was confirmed on transvaginal ultrasonographic examination, or endometriosis was found on laparoscopy during the infertility evaluation, but without surgical treatment, or endometriosis was previously diagnosed independently to infertility evaluation and was without treatment. Ultrasound diagnosis of endometriomas up to $3 \mathrm{~cm}$ as a round homogenous hypoechoic formation in the ovarian tissue, presence of less severe endometriosis with endometriotic focci in the pelvis, on uterus, uterine ligaments and ovaries and without tubal factor on diagnostic laparoscopy were the selection criteria for the Group II. Those patients were directly introduced to the IVF/ICSI.

EFI score was determined for all patients. If the total score is greater than 7, higher is the probability for spontaneous pregnancy. In this study EFI was used for the success assessment of the IVF/ICSI cycles.

\section{Endocrinological status}

On the third day of the menstrual cycle, before $\mathrm{COH}$, levels of follicle-stimulating hormone $(\mathrm{FSH})$, luteinizing hormone (LH), estradiol (E2) and progesterone (P4) were determined for all patients. AMH was determined for all patients independently to menstrual cycle. For the previously treated patients AMH was determined one month after surgery and prior to the additional medical treatment. For the patients without previous treatment of endometriosis $\mathrm{AMH}$ was determined prior to IVF/ICSI. FSH (IU/I), LH (IU/L), E2 (pg/mL), Pg ( $\mathrm{ng} / \mathrm{mL}$ ) values were determined by the chemiluminescent immunoassay (ECLIA, Access $2 \mathrm{im}$ munoassay system, Beckman Coulter). FSH/LH ratio was determined for each patient. Serum AMH $(n g / m L)$ values were determined by the enzyme-linked immunosorbent assay (ELISA, AMH II, Beckman Coulter) and were defined in interval ranges as low $(<1 \mathrm{ng} / \mathrm{mL})$, normal $(1-5 \mathrm{ng} / \mathrm{mL})$, and high (> $5 \mathrm{ng} / \mathrm{mL}$ ).

\section{IVF/ICSI procedure}

$\mathrm{COH}$ was performed according to three protocols: long protocol with GnRH agonist, short protocol with GnRH agonist and short protocol with GnRH antagonist.

The long protocol implied pituitary suppression with Diphereline ${ }^{\circledR}$, (Ferring Pharmaceuticals) at a dose of $0.1 \mathrm{mg}$ per day, for 7 days before the onset of the cycle and continued daily to the end of stimulation of ovulation. Short protocol implied pituitary suppression with GnRH agonist, tryptorelin at a dose of $0.1 \mathrm{mg}$ per day starting from the 2nd or 3rd day of the cycle, daily to the end of stimulation of ovulation. Short protocol with the GnRH antagonist implied the use of the use of $\mathrm{GnRH}$ antagonist cetrorelix (Cetrotide ${ }^{\circledR}$, Merck Serono) at a dose of $0.25 \mathrm{mg}$ per day from the sixth day 
of stimulation and continued daily to the end of stimulation. Ovarian stimulation started on day 2. or 3. of the cycle and was conducted with daily subcutaneus injections of FSH (follitropin $a-$ Gonal F ${ }^{\circledR}$, Merck Serono or follitropin $\beta$ - Puregon $^{\oplus}$, MSD) and/or HMG (menotropin - Menopur ${ }^{\circledR}$, Ferring Pharmaceuticals) with starting dose of 225 I.U. The ovarian stimulation was monitored by determining serum E2 and LH levels, as well as transvaginal ultrasonographic monitoring of follicular growth and endometrium thickness every second day from the day 6 of the cycle. When $\mathrm{E} 2$ values were above $400 \mathrm{pg} / \mathrm{mL}$ and there were at least two follicles greater than $18 \mathrm{~mm}$, choriogonadine was administered at a dose of 5000 to 10,000 IU. Follicular and oocyte aspiration was performed by transvaginal ultrasound control 34 to 36 hours after the administration of HCG. Selection of the protocols depended on the age, $\mathrm{EFI}, \mathrm{AMH}$ as well as AFC, FSH and E2 levels.

Ovarian response to stimulation was evaluated according to the number of retrieved oocytes, as poor ( $\leq 4$ oocytes), adequate (5-15 oocytes) and excessive (> 15 oocytes). Total number of embryos and their quality was assesed by embryologists and four classes of embryos were defined $A, B, C$ and $D$, where $A$ class represents the highest quality of embryos.

\section{Data collection}

Age, $\mathrm{BMI}, \mathrm{EFI}$, endometrioma presence, diameter and laterality, as well as the ASRM stage were determined for all patients in the defined groups prior to the IVF/ICSI treatment. Patients from the Group I were introduced to the IVF/ICSI after the endometriosis treatment, while patients from the Group II were directly introduced to the IVF/ICSI. The cycle outcomes were followed up, and afterwards cycles were analyzed depending on the outcome. Age, infertility duration, $\mathrm{BMI}, \mathrm{EFI}, \mathrm{ASRM}$ stage and score, hormone levels, the use of protocols for $\mathrm{COH}$, ovarian response and embryos were analysed in relation to the IVF/ICSI cycles outcome.

\section{Statistical analysis}

Descriptive statistics were used to summarize demographic, biochemical and clinical characteristics. Categorical variables were compared using Chi-square test. Continuous variables were compared by using ANOVA-test, or Kruskal-Wallis test. The association of selected variables with outcome was assessed with the Binary logistic regression analyses and determined the significance of the predictors. A significance of 0.05 was required for a variable to be included into the multivariate logistic regression model - Backward wild method, whereas 0.1 was the cut off value for exclusion. Odd ratios with the corresponding $95 \%$ confidence intervals were estimated. Analyses were performed using SPSS for Windows version 22 (SPSS, Inc, Chicago, IL).

\section{RESULTS \\ Patients}

The study included 73 patients who underwent 77 cycles of IVF/ICSI. The average patient's age was $34.14 \pm 3.53$ years, and average BMI was $22.55 \pm 2.45$. BMl lower than 25 had $64(87.7 \%)$ patients.

In the Group I there were 46 (63\%) patients, while in the Group II were 27 (37\%) patients. In the Group I 25 (54.3\%) patients had surgical treatment that was followed by the medical treatment, and 21 (45.7\%) patients were only surgically treated.

The description of the patients in the groups, prior to introduction into the IVF/ICSI procedures is shown in Table 1.The groups did not differ in any of the tested variables, except in the size of the endometriotic cyst, ASRM stage and the ASRM score of endometriosis. Significantly larger endometriomas $(p \leq 0.01)$ and higher ASRM scores $(p<0.01)$ were found in the subgroup of patients with combined (both surgical and medical) treatment compared to the subgroup of only surgically treated patients.

\section{Outcomes}

Description of all IVF/ICSI cycles in relation to the outcome is shown in Table 2 . In cycles with positive outcome (pregnancy), there were significantly more patients under 35 years $(71 \%)(p<0.044)$, infertility duration up to 3 years $(67.7 \%)(p<0.023)$, and with endometriosis that was previously treated $(77.4 \%)(p<0.039)$. EFI $>7$ was more frequent in the cases of pregnancy $(p<0.007)$. In these cases significantly lower basal FSH $(p<0.007), F S H / L H$ ratio $(p<0.002)$ were found, as well as significantly higher basal E2 ( $p<0.003)$, basal P4 ( $p<0.001)$ and AMH ( $p<0.000)$. Significantly lower doses of gonadotropins were needed in the cycles with achieved pregnancy $(p<0.028)($ Tab. 2$)$. The probability of success with long protocol with agonists was almost 3 times higher compared to the short protocols ( $\mathrm{OR}=2.98,95 \% \mathrm{Cl}$ 1.123 to 7.93$)$. Adequate ovarian response (58.1\%) was statistically significant $(p<0.008)$, more quality embryos were obtained $(90.3 \%)(p<0.001)$ and more clinical pregnancies $(77.4 \%)$ were achieved $(p<0.0001)$ in the group of cycles with positive outcome (Tab. 2).

Pregnancy rate per embriotransfer (PR/ET) was significantly higher in the Group I (44.19\%) compared to the Group II (21.74\%), ( $<<0.05)$. Subgroup A with combined therapy (both surgical and medical) had higher PR/ET (47.83\%) compared to the Group II (21.74\%), ( $<<0.05)$. Subgroup B (only surgical treatment) showed non-significantly elevated PR/ET (40.0\%) compared to the Group II. 
Table 1. Description of the IVF/ICSI patients in relation to the endometriosis therapy

\begin{tabular}{|c|c|c|c|c|c|c|}
\hline \multirow{2}{*}{\multicolumn{2}{|c|}{$x \pm S D$}} & \multicolumn{2}{|c|}{ Group I } & \multicolumn{2}{|c|}{ Group II } & \multirow{2}{*}{ p signif. } \\
\hline & & Median & $\mathrm{x} \pm \mathrm{SD}$ & Median & & \\
\hline \multicolumn{2}{|l|}{ Age (years) } & $33.88 \pm 3.20$ & 34 & $34.43 \pm 3.95$ & 36 & $\neq 0.507 / \mathrm{ns}$ \\
\hline \multicolumn{2}{|l|}{ BMI $\left[\mathrm{kg} / \mathrm{m}^{2}\right]$} & $22.66 \pm 2.44$ & 22.6 & $22.66 \pm 2.58$ & 22.7 & $\neq 0.996 / \mathrm{ns}$ \\
\hline \multicolumn{2}{|l|}{ EFI } & $6.04 \pm 1.96$ & 6 & $5.86 \pm 1.63$ & 6 & $\neq 0.676 / \mathrm{ns}$ \\
\hline \multicolumn{2}{|l|}{ FSH [IU/I] } & $7.43 \pm 3.78$ & 7.3 & $6.81 \pm 3.24$ & 6.55 & $+0.505 / \mathrm{ns}$ \\
\hline \multicolumn{2}{|l|}{ LH [IU/I] } & $3.99 \pm 2.37$ & 3.7 & $3.45 \pm 2.29$ & 3.3 & $+0.312 / \mathrm{ns}$ \\
\hline \multicolumn{2}{|l|}{ FSH/LH } & $2.32 \pm 1.41$ & 1.89 & $2.46 \pm 1.26$ & 2.06 & $+0.525 / \mathrm{ns}$ \\
\hline \multicolumn{2}{|l|}{ E2 [pg/mL] } & $41.75 \pm 21.13$ & 35 & $49.42 \pm 41.39$ & 35 & $+0.958 / \mathrm{ns}$ \\
\hline \multicolumn{2}{|l|}{ P4 [ng/mL] } & $1.05 \pm 0.92$ & 0.7 & $1.02 \pm 1.33$ & 0.56 & $+0.325 / \mathrm{ns}$ \\
\hline \multicolumn{2}{|l|}{ AMH ([ng/mL] } & $1.36 \pm 1.12$ & 0.9 & $1.54 \pm 1.15$ & 1.17 & $+0.164 / \mathrm{ns}$ \\
\hline \multirow{2}{*}{\multicolumn{2}{|c|}{ Cyst size [mm] }} & $56.5 \pm 13.54$ & 59 & $25.3 \pm 6.04$ & 22 & $\neq 0.0001^{* * *}$ \\
\hline & & \multicolumn{2}{|c|}{$\begin{array}{c}\text { Group I } \\
N=49[\%]\end{array}$} & \multicolumn{2}{|c|}{$\begin{array}{c}\text { Group II } \\
N=28[\%]\end{array}$} & p signif. \\
\hline \multirow{2}{*}{ EFI interval } & $\leq 7$ & \multicolumn{2}{|c|}{$31(63.3)$} & \multicolumn{2}{|c|}{$22(78.6)$} & \multirow{2}{*}{${ }^{*} 0.163 / \mathrm{ns}$} \\
\hline & $>7$ & \multicolumn{2}{|c|}{$18(36.7)$} & \multicolumn{2}{|c|}{$6(21.4)$} & \\
\hline \multirow{3}{*}{ Laterality } & Without cyst & \multicolumn{2}{|c|}{$2(4.1)$} & \multicolumn{2}{|c|}{$22(78.6)$} & \multirow{3}{*}{${ }^{*} 0.0001 * * *$} \\
\hline & Unilateral cyst & & & & & \\
\hline & Bilateral cysts & & & & & \\
\hline \multirow{2}{*}{ ASRM stage } & ASRM I/II & \multicolumn{2}{|c|}{$4(8.20)$} & \multicolumn{2}{|c|}{$13(46.4)$} & \multirow{2}{*}{${ }^{*} 0.002^{* *}$} \\
\hline & ASRM III/IV & & & & & \\
\hline \multirow{4}{*}{ ASRM score } & $<16$ & \multicolumn{2}{|c|}{$4(8.20)$} & \multicolumn{2}{|c|}{$13(46.4)$} & \\
\hline & $16-40$ & \multicolumn{2}{|c|}{$25(51.0)$} & & & $0 \cap 1 * * *$ \\
\hline & $41-70$ & & & & & 0.001 \\
\hline & $71+$ & & & & & \\
\hline
\end{tabular}

$(x \pm S D)-$ mean value \pm standard deviation, median, ${ }^{*}-$ Chi square $-x^{2}$ test, $\neq-F$ test, +- Kruskal Wallis test, $n s-p>0.05,{ }^{*} p<0.05,{ }^{* *} p<0.01,{ }^{* * *} p<0.001$

\section{ROC analysis}

The results of the $\mathrm{FSH} / \mathrm{LH}$ ratio $\mathrm{ROC}$ analysis was AUC $=0.711$, with a $95 \%$ confidence interval $\mathrm{Cl} 95 \%$ $0.594-0.828, p<0.002$ (Fig. 1). The FSH/LH ratio cut-off point of 1.99 was determined with sensitivity of $68 \%$, and specificity of $61 \%$.

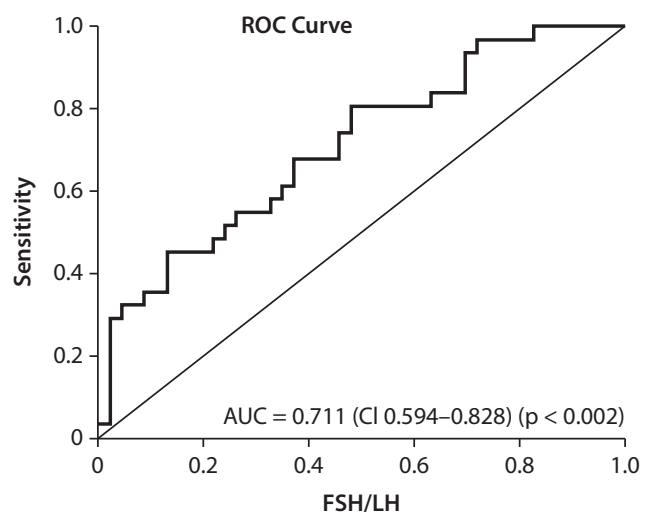

Figure 1. $\mathrm{FSH} / \mathrm{LH}$ ratio in relation to the outcome
The results of the AMH ROC analysis was AUC $=0.795$, with a $95 \%$ confidence interval Cl 95\% 0.683-0.907, $\mathrm{p}<0.0001$ (Fig. 2). The AMH cut-off point of 0.89 was determined with sensitivity of $81 \%$, and specificity of $50 \%$.

Successful IVF/ICSI outcomes were more frequent when: $\mathrm{FSH}<7 \mathrm{IU} / \mathrm{I}(74.2 \%, \mathrm{p}<0.001), \mathrm{FSH} / \mathrm{LH}<2(67.7 \%, \mathrm{p}<0.023)$,

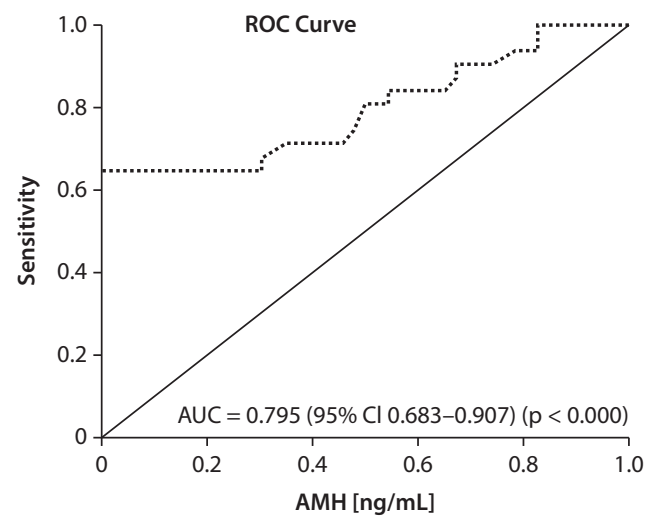

Figure 2. AMH in relation to the outcome 
Table 2. Description of the IVF/ICSI cycles in relation to the outcome

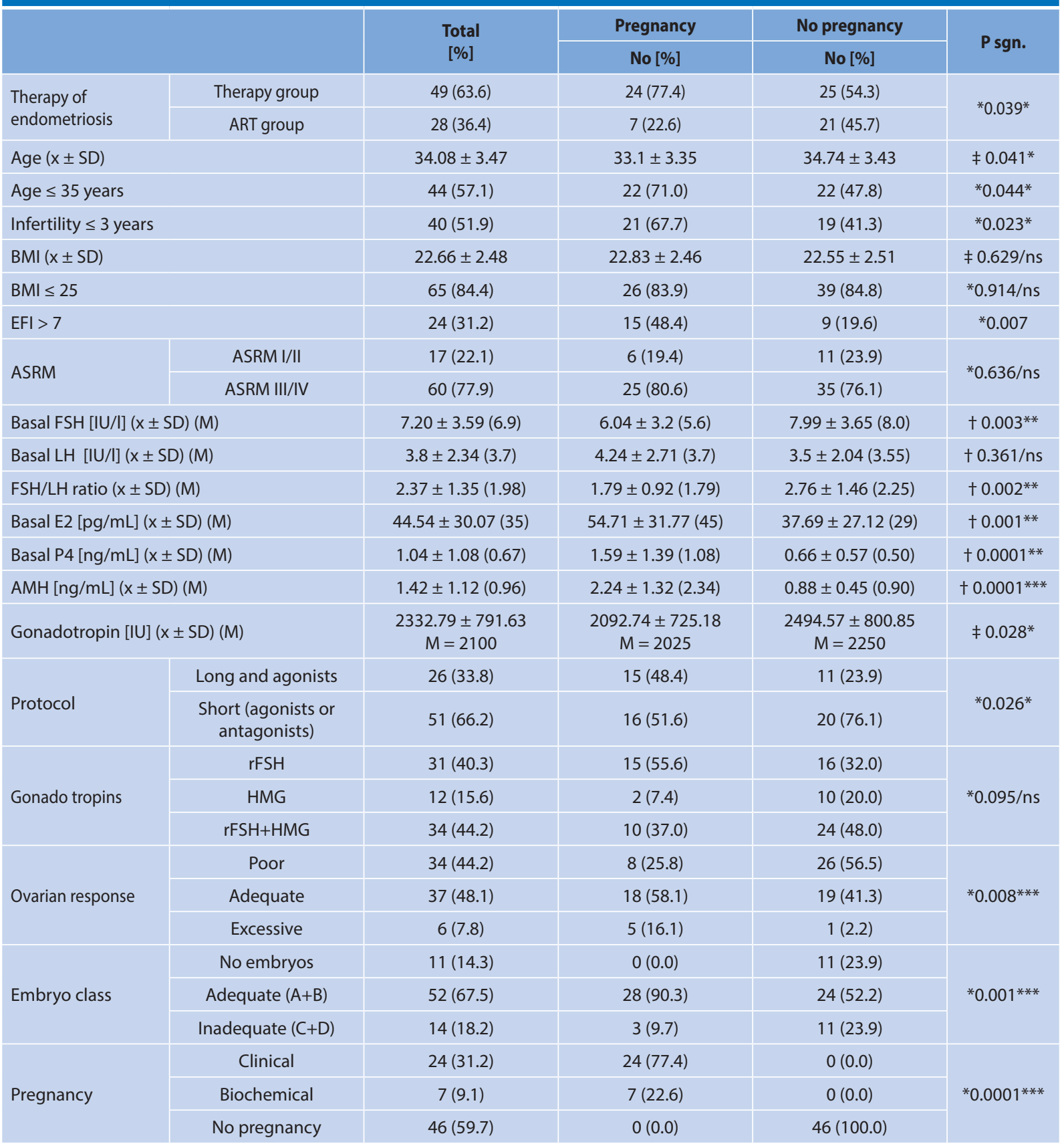

$(x \pm S D)-$ mean value \pm standard deviation, $(M)-$ median, ${ }^{*}-x^{2}$ test, $\neq-F$ test, $+-K-W$ test, ns $-p>0.05,{ }^{*} p<0.05,{ }^{* *} p<0.01,{ }^{* * *} p<0.001$

E2 $\geq 30 \mathrm{pg} / \mathrm{mL}$ (77.4\%, $\mathrm{p}<0.009), \mathrm{P} 4 \geq 0.7 \mathrm{ng} / \mathrm{mL}$ (71.0\%, $\mathrm{p}<0.0001), \mathrm{AMH} \geq 0.9 \mathrm{ng} / \mathrm{mL}(80.6 \%, \mathrm{p}<0.006)($ Tab. 3).

\section{Univariate and multivariate logistic regression analysis}

Table 4 represents univariate and multivariate logistic regression model for the outcomes. Predictors of the successful IVF/ICSI outcome in multivariate logistic regression model were: A class of embryos (OR $=41.749,95 \%$ $\mathrm{Cl} 41.749-383.61), \mathrm{AMH} \geq 0.9 \mathrm{ng} / \mathrm{mL}(\mathrm{OR}=22.74,95 \% \mathrm{Cl}$
1.2.176-237.68), $\mathrm{P} 4 \geq 0.7 \mathrm{ng} / \mathrm{mL}(\mathrm{OR}=6.429,95 \% \mathrm{Cl} 1.195-$ $-34.593)$, previous therapy of endometriosis ( $O R=5.241$, $95 \% \mathrm{Cl} 1.598-17.193)$, and the use of the long protocol with agonists (OR $=5.796,95 \% \mathrm{Cl} 1.007-33.349)$.

\section{DISCUSSION}

Pregnancies following IVF/ICSI were mostly achieved in patients under 35 years with infertility duration up to 3 years. EFI score associated anamnestic and clinical data very well and has proven to be a good predictor of the 
Table 3. Applied Cut-off values of hormones in relation to the outcome

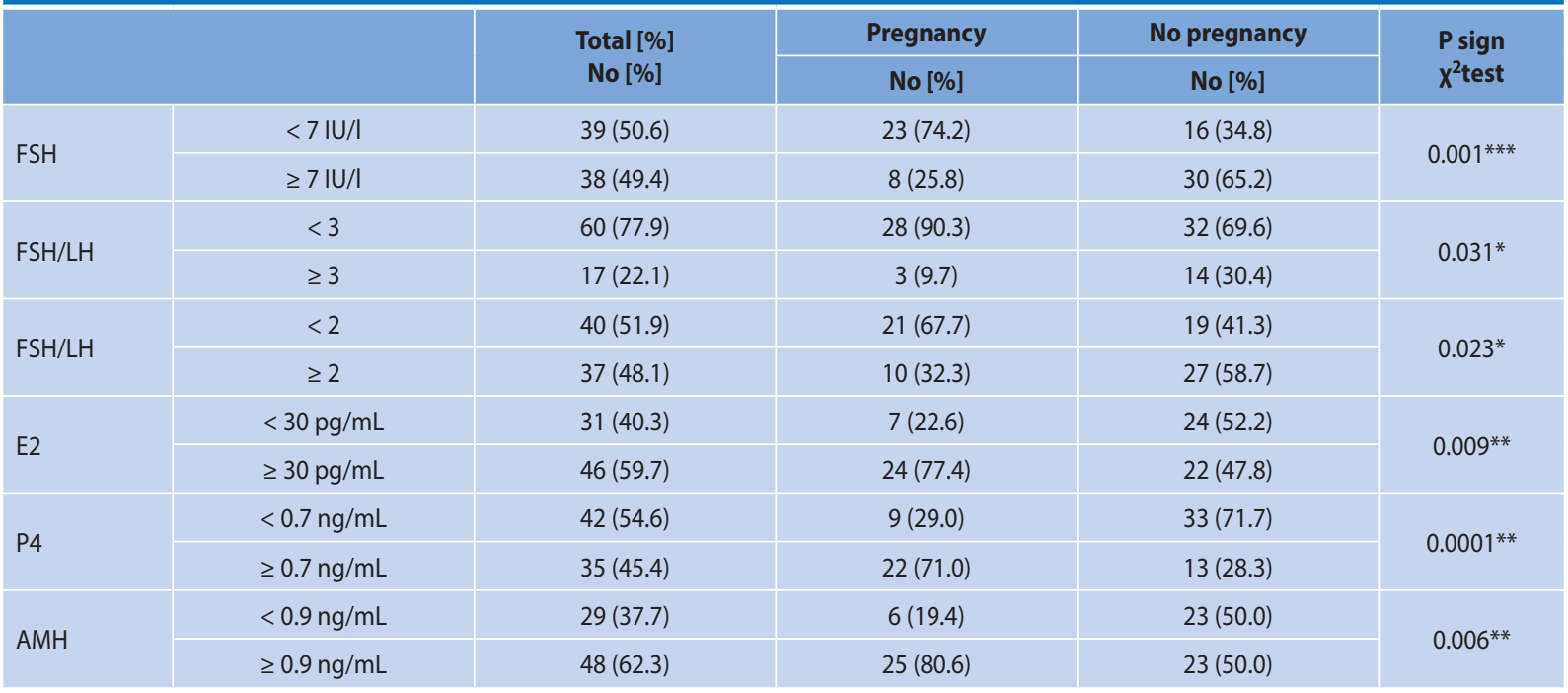

$\mathrm{X}^{2}$ test, ns $-p>0.05,{ }^{*} p<0.05,{ }^{* *} p<0.01,{ }^{* * *} p<0.001$

Table 4. Binary logistic regression analysis (LRA) for the outcomes

\begin{tabular}{|c|c|c|c|c|c|c|c|c|}
\hline & \multicolumn{4}{|c|}{ Univariate LRA } & \multicolumn{4}{|c|}{ Multivariate LRA } \\
\hline & \multirow{2}{*}{ OR } & \multirow{2}{*}{ Sig. } & \multicolumn{2}{|c|}{ 95\% C.I. } & \multirow{2}{*}{ OR } & \multirow{2}{*}{ Sig. } & \multicolumn{2}{|c|}{$95 \%$ C.I. } \\
\hline & & & Lower & Upper & & & Lower & Upper \\
\hline Therapy & 1.697 & 0.043 & 1.018 & 2.830 & 5.241 & 0.006 & 1.598 & 17.193 \\
\hline Age $\leq 35$ & 2.667 & 0.047 & 1.013 & 7.017 & & & & \\
\hline Primary infertility $\leq 3$ & 2.984 & 0.025 & 1.149 & 7.753 & & & & \\
\hline $\mathrm{EFI}>7$ & 3.854 & 0.009 & 1.399 & 10.617 & & & & \\
\hline Endometrioma presence & 2.679 & 0.071 & 0.919 & 7.808 & & & & \\
\hline $\mathrm{FSH} / \mathrm{LH}<2$ & 2.984 & 0.025 & 1.151 & 7.753 & & & & \\
\hline $\mathrm{E} 2 \geq 30 \mathrm{pg} / \mathrm{mL}$ & 3.740 & 0.011 & 1.347 & 10.388 & & & & \\
\hline $\mathrm{P} 4 \geq 0.7 \mathrm{ng} / \mathrm{mL}$ & 6.205 & 0.0001 & 2.268 & 16.980 & 6.429 & 0.030 & 1.195 & 34.593 \\
\hline $\mathrm{AMH} \geq 0.9 \mathrm{ng} / \mathrm{mL}$ & 4.167 & 0.008 & 1.441 & 12.051 & 22.740 & 0.009 & 2.176 & 237.68 \\
\hline Aspirated oocytes $>5$ & 3.582 & 0.009 & 1.369 & 9.375 & & & & \\
\hline Adequate/excessive ovarian response & 3.737 & 0.003 & 1.384 & 10.094 & & & & \\
\hline Obtained embryos $>2$ & 3.272 & 0.014 & 1.266 & 8.460 & & & & \\
\hline A class of embryos & 6.429 & 0.0001 & 2.277 & 18.147 & 41.749 & 0.001 & 4.544 & 383.61 \\
\hline B class of embryos & 3.136 & 0.024 & 1.164 & 8.448 & & & & \\
\hline Long protocol & 2.983 & 0.023 & 1.123 & 7.927 & 5.796 & 0.049 & 1.007 & 33.349 \\
\hline Gonadotropins doses < $2000 \mathrm{IU}$ & 2.656 & 0.047 & 1.013 & 6.965 & & & & \\
\hline
\end{tabular}

successful treatment. It is well known that superior variable in clinical pregnancy prediction is the patient's age, but several hormones can predict oocyte count in different age groups as well [10]. FSH is increasing, while $\mathrm{AMH}$ is decreasing with age, and some studies point out that $\mathrm{AMH}$ is a more reliable biomarker compared to FSH [11]. The AMH level well correlates with other markers of ovarian reserve, such as antral follicle count (AFC) or day 3 serum FSH, and can predict ovarian responsiveness to $\mathrm{COH}$ [12]. It is widely quoted that $\mathrm{AMH}$ has equal sensitivity and specificity as $\mathrm{AFC}$, and is better than $\mathrm{FSH}, \mathrm{E2}, \mathrm{LH}, \mathrm{FSH} / \mathrm{LH}$ ratio or inhibin-B levels [13].

Pregnancies were achieved in all ASRM stages of endometriosis equally. Higher stages of endometriosis had previously determined the therapy selection, thus endometriosis as a chronic disease was suppressed and micro 
conditions for achieving pregnancy were established. We found that PR/ET was significantly higher in the Group I compared to the Group II, as well as that PR/ET was higher in the Subgroup A compared to the Group II. This suggests that previous endometriosis therapy had positive influence on the IVF/ICSI outcome, especially in the cases of severe endometriosis that had been treated with combined, both surgical and medical therapy. In multivariate logistic regression the therapy for endometriosis was found to be a predictive factor for pregnancy after IVF/ICSI.

Studies report that endometriosis presence does not affect ovarian response to exogenous gonadotropin stimulation when the ovarian reserve is not significantly impaired [14]. Ovarian reserve could be compromised after previous endometriosis surgical treatment. In our study it might be that additional medical therapy preserved ovarian reserve and at the same time suppressed pathophysiological pathways that affect oocyte maturation, fertilization and implantation.

Even though FSH, as mostly used ovarian marker could be normal, some patients might respond poorly to $\mathrm{COH}$ [15]. Elevated $\mathrm{FSH} / \mathrm{LH}$ ratio $\geq 2$ is strongly related to the reduced ovarian response to stimulation and lower pregnancy rates in IVF treatment [16]. In the cases of achieved pregnancy, we found significantly lower basal FSH, FSH/LH ratio, as well as significantly higher basal E2, basal P4 and $\mathrm{AMH}$ with less gonadotropins spent than in cases with no pregnancy. Yoo et al. showed that serum AMH level in women with endometriosis had a positive correlation with the number of retrieved oocytes and the mature oocytes, while there was no correlation between serum FSH and number of mature oocytes, suggesting that AMH level might be a better marker than serum FSH or age for predicting the number of retrieved oocytes [6]. Similar findings were found in our study as well. Bilaterality of the endometriomas also represents a significant factor for AMH decline after surgery [17].

The presence of endometrioma does not reduce the number of retrieved oocytes in a $\mathrm{COH}$ cycle for IVF, but the ovarian response could be affected by the size of endometriomas, bilaterality, previous surgeries, recurrence, and the patient's age [18]. Also there are studies suggesting that patients age and cyst diameter were not related to the extent of $\mathrm{AMH}$ decline, while the excision of bilateral endometriomas might be associated with a greater loss of ovarian reserve $[13,19]$. In addition levels of $\mathrm{AMH}$ declined more in patients with endometriomas than in patients with other benign ovarian cysts [13]. In women with endometriosis, the ORs of having AMH levels less than $1 \mathrm{ng} / \mathrm{mL}$ are significantly increased both in advanced age and in the cases of a previous surgery as well, making it independent factor for diminishing ovarian reserve [20]. Ovarian reserve damage due to surgical treatment of endometriomas further requires significantly higher gonadotrophins doses, longer ovarian stimulation and usually has higher cancelation rate for poor response [21]. However, after the prolonged down-regulation with $\mathrm{GnRH}$ agonists following the operation, every 4 weeks for 3 months, size of the growing follicles appears to be normal, which is indicated by normal $\mathrm{AMH}$ levels 4 and 8 weeks after the therapy [22]. It could be recommended that when the lower ovarian response is expected, AMH serum level should be measured previous to a $\mathrm{COH}$ to optimise the dosage of gonadotropin treatment.

Decreased ovarian response and lower pregnancy rates, as well as higher cycle cancellation rate are expected when $\mathrm{FSH} / \mathrm{LH}$ ratio is elevated in the presence of a normal basal FSH [23]. Poor IVF outcomes even with FSH/LH ratios $\geq 2$ were found, despite the use of aggressive protocols and higher doses of ovarian stimulation drugs [24]. FSH/LH ratio is mostly correlated with the lower $\mathrm{LH}$ level and has only a small correlation with the FSH level [24].

Higher concentrations of peritoneal fluid in patients with minimal/mild endometriosis may contain factors that compromise ovarian steroidogenesis and reduce $\mathrm{P} 4$ release [25]. Low progesterone may alter fine balance between metalloproteinases and tissue inhibitors, thus demanding its supplementation in women with endometriosis undergoing IVF [26]. As recent studies have shown, in endometriosis we can expect higher concentration of progesterone (PR) and estrogen receptors (ER) on granulosa cells [27]. When the endometriosis is treated, expression of the PR on granulosa cells might be lower, thus serum P4 levels may be higher compared to those with non-treated endometriosis. Higher expression of PR on granulosa cell may lead to inadequate follicular maturation, obtaining low quality oocytes and embryos. Quality embryos can be retrieved from granulosa cells with lower PR expression, so the reduction of PR stimulated by LH might regulate oocyte maturity [27].

The small number of retrieved oocytes, mature oocytes, and lower pregnancy rates were found in cases with increased $\mathrm{FSH} / \mathrm{LH}$ ratio $\geq 2$, when GnRH antagonists were administered [28]. Long protocol with agonists was most frequently used protocol in the cycles with achieved pregnancy. Studies show that $\mathrm{GnRH}$-agonist protocol may result in higher pregnancy as well as live-birth rates after fresh ET in women with endometriosis [29]. It is possible that protocols with antagonists might negatively impact endometrial receptivity in these situations [29]. The role of LH supplementation for improving outcomes in patients undergoing $\mathrm{COH}$ for IVF cycles is unclear, although it has been suggested that patients with poor ovarian reserve may benefit from LH supplementation [30]. In our study significantly higher AMH in the cycles with achieved pregnancy shows adequate ovarian reserve. More gonadotropins were used in the Group II in the cycles with reduced ovarian reserve that were followed by unsuccessful 
outcomes. In this group we frequently more used rFSH and HMG, which is the most common choice in the situations where a worse ovarian response is expected. The use of HMG in patients with high basal $\mathrm{FSH} / \mathrm{LH}$, may be associated with significantly higher number of top-quality embryos, higher implantation and clinical pregnancy rates when compared with the use of $\mathrm{rFSH}$ [31]. In the pregnancy achieved cycles we used $\mathrm{rFSH}$ in $55.6 \%$, and the combination of FSH and HMG in $37 \%$ of cycles. We found no difference between the use of rFSH and HMG between the groups. Additional treatment with GnRH agonist before IVF-ET or ultralong GnRHa therapy has been reported to improve the outcome of IVF/ET in endometriosis, possibly due to reduction of the detrimental effects of cytotoxic cytokines and oxidative stress in the ovary in these patients [32]. Hence, it might be suggested to continue to IVF/ICSI procedures immediately after the endometriosis treatment especially if the $\mathrm{GnRH}$ agonists are used.

Ballester M. in his study pointed up that covariates such as patients age, serum AMH level, presence of DIE and number of IVF/ICSI cycles were clinically significant in the pregnancy rate prediction after IVF/ICSI cycles in patients with endometriosis [33]. Patients with serum AMH levels of $0.6 \mathrm{ng} / \mathrm{mL}$ or above had twice the number of oocytes retrieved, a greater number of day 3 embryos and a higher clinical pregnancy rate compared with patients with lower $\mathrm{AMH}$ levels [33]. In the multivariate logistic regression analysis we found that predictors for the successful IVF/ICSI outcome were: previous therapy for endometriosis, $\mathrm{AMH} \geq 0.9 \mathrm{ng} / \mathrm{mL}$, $\mathrm{P} 4 \geq 0.7 \mathrm{ng} / \mathrm{mL}$, the use of the long protocol with agonists, and the $A$ class of embryos.

\section{CONCLUSIONS}

Endometriosis should be treated before attempting IVF/ICSI either surgically or with medical therapy. Special care must be taken over preserving ovarian reserve, whose superior marker is AMH. Preserved ovarian reserve is the basis of good response to stimulation, and the use of long protocol with agonists could be advised.

\section{Conflict of interest}

The authors report no conflicts of interest.

\section{REFERENCES}

1. Kokcu A. Possible effects of endometriosis-related immune events on reproductive function. Arch Gynecol Obstet. 2013; 287(6): 1225-1233, doi: 10.1007/s00404-013-2767-2, indexed in Pubmed: 23430032.

2. Sanchez AM, Vanni VS, Bartiromo L, et al. Is the oocyte quality affected by endometriosis? A review of the literature. J Ovarian Res. 2017; 10(1): 43, doi: 10.1186/s13048-017-0341-4, indexed in Pubmed: 28701212.

3. Menezo YJR, Silvestris E, Dale B, et al. Oxidative stress and alterations in DNA methylation: two sides of the same coin in reproduction. Reprod Biomed Online. 2016; 33(6): 668-683, doi: 10.1016/j.rbmo.2016.09.006, indexed in Pubmed: 27742259.

4. Schleedoorn MJ, Nelen WL, Dunselman GAJ, et al. EndoKey Group, European Society of Human Reproduction and Embryology. ESHRE guideline: management of women with endometriosis. Hum Reprod. 2014; 29(3): 400-412, doi: 10.1093/humrep/det457, indexed in Pubmed: 24435778.

5. Practice Committee of the American Society for Reproductive Medicine. Endometriosis and infertility: a committee opinion. Fertil Steril. 2012; 98(3): 591-598, doi: 10.1016/j.fertnstert.2012.05.031, indexed in Pubmed: 22704630.

6. Yoo $\mathrm{JiH}, \mathrm{Cha} \mathrm{SH}$, Park CW, et al. Serum anti-Müllerian hormone is a better predictor of ovarian response than $\mathrm{FSH}$ and age in IVF patients with endometriosis. Clin Exp Reprod Med. 2011; 38(4): 222-227, doi: 10.5653/cerm.2011.38.4.222, indexed in Pubmed: 22384446.

7. Nelson SM, Yates RW, Lyall H, et al. Anti-Müllerian hormone-based approach to controlled ovarian stimulation for assisted conception. Hum Reprod. 2009; 24(4): 867-875, doi: 10.1093/humrep/den480, indexed in Pubmed: 19136673.

8. Broer SL, Broekmans FJM, Laven JSE, et al. Anti-Müllerian hormone: ovarian reserve testing and its potential clinical implications. Hum Reprod Update. 2014; 20(5): 688-701, doi: 10.1093/humupd/dmu020, indexed in Pubmed: 24821925.

9. Adamson GD, Pasta DJ. Endometriosis fertility index: the new, validated endometriosis staging system. Fertil Steril. 2010; 94(5): 1609-1615, doi: 10.1016/j.fertnstert.2009.09.035, indexed in Pubmed: 19931076.

10. Shahrokh Tehraninezhad E, Mehrabi F, Taati R, et al. Analysis of ovarian reserve markers ( $\mathrm{AMH}, \mathrm{FSH}, \mathrm{AFC}$ ) in different age strata in IVF/ICSI patients. Int J Reprod Biomed (Yazd). 2016; 14(8): 501-506, doi: 10.29252/ijrm.14.8.501, indexed in Pubmed: 27679824.

11. Barbakadze L, Kristesashvili J, Khonelidze N, et al. The correlations of anti-mullerian hormone, follicle-stimulating hormone and antral follicle count in different age groups of infertile women. Int J Fertil Steril. 2015; 8(4): 393-398, indexed in Pubmed: 25780521.

12. Somigliana E, Berlanda N, Benaglia L, et al. Surgical excision of endometriomas and ovarian reserve: a systematic review on serum antimüllerian hormone level modifications. Fertil Steril. 2012; 98(6): 1531-1538, doi: 10.1016/j.fertnstert.2012.08.009, indexed in Pubmed: 22975114.

13. Chen $\mathrm{Y}$, Pei H, Chang Y, et al. The impact of endometrioma and laparoscopic cystectomy on ovarian reserve and the exploration of related factors assessed by serum anti-Mullerian hormone: a prospective cohort study. J Ovarian Res. 2014; 7: 108, doi: 10.1186/s13048-014-0108-0, indexed in Pubmed: 25424986.

14. Luca A, Nemescu D, Butnaru M, et al. Ovarian stimulation outcome in infertile women with endometriosis undergoing IVF. Ginekol Pol. 2016; 87(1): 37-41, doi: 10.17772/gp/60073, indexed in Pubmed: 27306467.

15. Seckin B, Turkcapar F, Ozaksit G. Elevated day 3 FSH/LH ratio: a marker to predict IVF outcome in young and older women. J Assist Reprod Genet. 2012; 29(3): 231-236, doi: 10.1007/s10815-011-9695-5, indexed in Pubmed: 22183503.

16. Prasad S, Gupta T, Divya A. Correlation of the Day 3 FSH/LH Ratio and LH Concentration in Predicting IVF Outcome. J Reprod Infertil. 2013; 14(1): 23-28, indexed in Pubmed: 23926557.

17. Kwon SuK, Kim SH, Yun SC, et al. Decline of serum antimüllerian hormone levels after laparoscopic ovarian cystectomy in endometrioma and other benign cysts: a prospective cohort study. Fertil Steril. 2014; 101(2): 435-441, doi: 10.1016/j.fertnstert.2013.10.043, indexed in Pubmed: 24290000.

18. Ferrero S, Scala C, Tafi E, et al. Impact of large ovarian endometriomas on the response to superovulation for in vitro fertilization: A retrospective study. Eur J Obstet Gynecol Reprod Biol. 2017; 213:17-21, doi: 10.1016/j. ejogrb.2017.04.003, indexed in Pubmed: 28407512.

19. Uncu G, Kasapoglu I, Ozerkan K, et al. Prospective assessment of the impact of endometriomas and their removal on ovarian reserve and determinants of the rate of decline in ovarian reserve. Hum Reprod. 2013; 28(8): 2140-2145, doi: 10.1093/humrep/det123, indexed in Pubmed: 23624580.

20. Streuli I, de Ziegler D, Gayet V, et al. In women with endometriosis anti-Müllerian hormone levels are decreased only in those with previous endometrioma surgery. Hum Reprod. 2012; 27(11): 3294-3303, doi: 10.1093/humrep/des274, indexed in Pubmed: 22821432.

21. Roustan A, Perrin J, Debals-Gonthier M, et al. Surgical diminished ovarian reserve after endometrioma cystectomy versus idiopathic DOR: comparison of in vitro fertilization outcome. Hum Reprod. 2015; 30(4): 840-847, doi: 10.1093/humrep/dev029, indexed in Pubmed: 25740883.

22. Mohamed KA, Davies WAR, Lashen $\mathrm{H}$. Antimüllerian hormone and pituitary gland activity after prolonged down-regulation with goserelin acetate. Fertil Steril. 2006; 86(5): 1515-1517, doi: 10.1016/j. fertnstert.2006.03.047, indexed in Pubmed: 16978621. 
23. Orvieto R, Meltzer S, Rabinson J, et al. Does day 3 luteinizing-hormone level predict IVF success in patients undergoing controlled ovarian stimulation with GnRH analogues? Fertil Steril. 2008; 90(4): 1297-1300, doi: 10.1016/j.fertnstert.2007.10.058, indexed in Pubmed: 18249369.

24. Liu KE, Greenblatt EM. Elevated day 3 follicle-stimulating hormone/luteinizing hormone ratio $>$ or $=2$ is associated with higher rates of cancellation in in vitro fertilization-embryo transfer cycles. Fertil Steril. 2008; 90(2): 297-301, doi: 10.1016/j.fertnstert.2007.06.038, indexed in Pubmed: 18023434.

25. Gomes FM, Navarro PA, de Abreu LG, et al. Effect of peritoneal fluid from patients with minimal/mild endometriosis on progesterone release by human granulosa-lutein cells obtained from infertile patients without endometriosis: a pilot study. Eur J Obstet Gynecol Reprod Biol. 2008; 138(1): 60-65, doi: 10.1016/j.ejogrb.2007.12.008, indexed in Pubmed: 18276059.

26. Singh AK, Chattopadhyay R, Chakravarty B, et al. Altered circulating levels of matrix metalloproteinases 2 and 9 and their inhibitors and effect of progesterone supplementation in women with endometriosis undergoing in vitro fertilization. Fertil Steril. 2013; 100(1): 127-34.e1, doi: 10.1016/j.fertnstert.2013.03.006, indexed in Pubmed: 23557756.

27. Karita M, Yamashita Y, Hayashi A, et al. Does advanced-stage endometriosis affect the gene expression of estrogen and progesterone receptors in granulosa cells? Fertil Steril. 2011; 95(3): 889-894, doi: 10.1016/j. fertnstert.2010.12.026, indexed in Pubmed: 21269613.

28. Lyu SW, Kim JiW, Choi CH, et al. Impact of high basal FSH/LH ratio in women with normal FSH levels on in vitro fertilization outcomes. Gynecol
Endocrinol. 2013; 29(5):424-429, doi: 10.3109/09513590.2012.743002, indexed in Pubmed: 23544714.

29. Kolanska K, Cohen J, Bendifallah S, et al. Pregnancy outcomes after controlled ovarian hyperstimulation in women with endometriosis-associated infertility: GnRH-agonist versus GnRH-antagonist. J Gynecol Obstet Hum Reprod. 2017; 46(9): 681-686, doi: 10.1016/j.jogoh.2017.09.007, indexed in Pubmed: 28970135.

30. Vuong TNL, Phung HT, Ho MT. Recombinant follicle-stimulating hormone and recombinant luteinizing hormone versus recombinant follicle-stimulating hormone alone during $\mathrm{GnRH}$ antagonist ovarian stimulation in patients aged $\geq 35$ years: a randomized controlled trial. Hum Reprod. 2015; 30(5): 1188-1195, doi: 10.1093/humrep/dev038, indexed in Pubmed: 25740882.

31. Orvieto R, Homburg R, Meltcer S, et al. HMG improves IVF outcome in patients with high basal FSH/LH ratio: a preliminary study. Reprod Biomed Online. 2009; 18(2): 205-208, doi: 10.1016/s1472-6483(10)60257-x, indexed in Pubmed: 19192340.

32. Tamura H, Takasaki A, Nakamura Y, et al. A pilot study to search possible mechanisms of ultralong gonadotropin-releasing hormone agonist therapy in IVF-ET patients with endometriosis. J Ovarian Res. 2014; 7: 100, doi: 10.1186/s13048-014-0100-8, indexed in Pubmed: 25331066.

33. Ballester M, Oppenheimer A, d'Argent EM, et al. Nomogram to predict pregnancy rate after ICSI-IVF cycle in patients with endometriosis. Hum Reprod. 2012; 27(2): 451-456, doi: 10.1093/humrep/der392, indexed in Pubmed: 22114107. 